ARTICLE

Received 5 Nov 2013 | Accepted 2 Jan 2014 | Published 24 Jan $2014 \quad$ DOI: 10.1038/ncomms4186

\title{
Effect of defects on the intrinsic strength and stiffness of graphene
}

\author{
Ardavan Zandiatashbar ${ }^{1, \star}$, Gwan-Hyoung Lee ${ }^{2,3, \star}$, Sung Joo An², Sunwoo Lee ${ }^{4}$, Nithin Mathew ${ }^{1}$, \\ Mauricio Terrones $5,6,7$, Takuya Hayashi ${ }^{8}$, Catalin R. Picu' ${ }^{1}$, James Hone ${ }^{2}$ \& Nikhil Koratkar 1,9
}

It is important from a fundamental standpoint and for practical applications to understand how the mechanical properties of graphene are influenced by defects. Here we report that the two-dimensional elastic modulus of graphene is maintained even at a high density of $s p^{3}$-type defects. Moreover, the breaking strength of defective graphene is only 14\% smaller than its pristine counterpart in the $s p^{3}$-defect regime. By contrast, we report a significant drop in the mechanical properties of graphene in the vacancy-defect regime. We also provide a mapping between the Raman spectra of defective graphene and its mechanical properties. This provides a simple, yet non-destructive methodology to identify graphene samples that are still mechanically functional. By establishing a relationship between the type and density of defects and the mechanical properties of graphene, this work provides important basic information for the rational design of composites and other systems utilizing the high modulus and strength of graphene.

\footnotetext{
${ }^{1}$ Department of Mechanical, Aerospace and Nuclear Engineering, Rensselaer Polytechnic Institute, Troy, New York 12180, USA. ${ }^{2}$ Department of Mechanical Engineering, Columbia University, New York, New York 10027, USA. ${ }^{3}$ Department of Materials Science and Engineering, Yonsei University, Seoul 120-749, Korea. ${ }^{4}$ Department of Electrical Engineering, Columbia University, New York, New York 10027, USA. ${ }^{5}$ Department of Physics, The Pennsylvania State University, University Park, Pennsylvania 16802, USA. ${ }^{6}$ Department of Chemistry, The Pennsylvania State University, University Park, Pennsylvania 16802, USA. ${ }^{7}$ Department of Materials Science and Engineering and Center for 2-Dimensional and Layered Materials, The Pennsylvania State University, University Park, Pennsylvania 16802, USA. ${ }^{8}$ Faculty of Engineering and Research Center for Exotic Nanocarbons, Shinshu University, 4-17-1 Wakasato, Nagano 380-8553, Japan. ${ }^{9}$ Department of Materials Science and Engineering, Rensselaer Polytechnic Institute, Troy, New York 12180, USA. * These authors contributed equally to this work. Correspondence and requests for materials should be addressed to C.R.P. (email: picuc@rpi.edu) or to J.H. (email: jh2228@columbia.edu) or to N.K. (email: koratn@rpi.edu).
} 


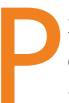
ristine (that is, defect-free) graphene exhibits ultra-high elastic modulus and unsurpassed strength ${ }^{1}$. Atomic force microscope (AFM)-based nanoindentation experiments performed on defect-free graphene has yielded a Young's modulus of $\sim 1 \mathrm{TPa}$ and tensile strength of over $100 \mathrm{GPa}$ (refs $2,3)$, as theoretically predicted ${ }^{1}$. However, for various practical applications of graphene, such as composites ${ }^{4-6}$, chemical sensors ${ }^{7,8}$, ultra capacitors 9 , transparent electrodes ${ }^{10,11}$, photovoltaic cells ${ }^{12}$ and biodevices ${ }^{13-15}$, the emergence of defects in the graphene lattice is inevitable either because of the production process used ${ }^{16-18}$ or because of the environmental and operating conditions under which the graphene device operates $^{19-23}$. In addition, in biodevices and DNA-decorated graphene ${ }^{13-15}$, the presence of defects is essential for the desired functionality. Defect engineering of graphene is also used in nanoelectronics for opening a band gap ${ }^{24}$, DNA-sequencing through nanopores ${ }^{25}$ and selective molecular sieving through the nanopores of suspended graphene ${ }^{26}$. Given that defects are ubiquitous in the operational environment of graphene devices, it is important to understand how the defectiveness of graphene has an impact on its elastic properties and intrinsic strength.

Computational work has investigated the mechanical properties of graphene in the presence of vacancies ${ }^{27-31}$, bond reconstruction $^{32-35}$ and functional groups ${ }^{36}$. Several studies have also shown the formation and structural evolution of defects in graphene using both experimental ${ }^{37,38}$ and theoretical tools ${ }^{39-42}$. However, on the experimental side, there has been very limited study of how the density and type of defects affect the mechanical properties of graphene. For instance, although the elastic stiffness of graphene oxide (GO), which represents a highly defective state of graphene, was reported by AFM observation of shape changes in GO membranes ${ }^{43}$, only its elastic modulus was provided, without measurement of breaking strength.

In this study, we use a modified oxygen plasma technique to induce defects in pristine graphene in a controlled manner. We utilize Raman spectroscopy and transmission electron microscopy (TEM) to characterize the defects. We then use AFM nanoindentation to experimentally quantify the stiffness and strength of defective graphene, and use finite element modelling and molecular simulations to understand this behaviour. Our results indicate that graphene sheets with defects are more rugged and structurally robust than what was previously thought. While thermal and electrical transport in graphene is very sensitive to disruptions in the $s p^{2}$-bonding network, its mechanical properties are far more tolerant of defects and imperfections.

\section{Results}

Sample preparation. In our experimental study, a $1 \times 1 \mathrm{~cm}^{2}$ array of circular wells with diameters ranging from 0.5 to $5 \mu \mathrm{m}$ was patterned on a $\mathrm{Si}$ chip with a $300-\mathrm{nm} \mathrm{SiO}_{2}$-capping layer, by photolithography and reactive ion etching. Graphene was mechanically exfoliated on the patterned substrate $e^{2,21}$ to create suspended membranes. Monolayer graphene flakes were identified by optical microscopy and Raman spectroscopy. Elastic stiffness and breaking force were measured by AFM nanoindentation with a diamond AFM tip, as shown in the schematic of Fig. 1a. Figure 1b shows optical micrograph of monolayer and bilayer graphene sheets covering distinct holes of different diameters. Before the indentation testing, non-contact mode AFM imaging was used to confirm that the graphene sheets are well suspended as shown in Fig. 1c.

Introduction of defects. A tabletop oxygen plasma etcher was used to induce defects in the suspended graphene sheets (see Methods). Weak oxidation by ion bombardment, oxygen plasma or ultraviolet irradiation has been used to etch graphene or generate defects ${ }^{26,44-46}$. Compared with these techniques, the etch rate of graphene in the oxygen plasma is fast: $\sim 9$ layers per minute at a chamber pressure of $\sim 215$ mTorr. To shield the graphene from direct exposure to the plasma, the graphenedeposited Si chips were placed upside down in the plasma chamber between two glass slides (see Supplementary Fig. 1a,b). This reduced the etch rate by a factor of $\sim 30$ (Supplementary Fig. 1c,d), and allowed the suspended graphene to survive without breaking or collapse even after plasma exposure of $55 \mathrm{~s}$.

Raman spectroscopy. To quantitatively examine the type and density of defects in the graphene sheets, the graphene was characterized using Raman spectroscopy ${ }^{47,48}$. Figure 2 a shows the evolution of the Raman spectrum with increasing plasma time for a typical monolayer graphene supported on the $\mathrm{SiO}_{2} / \mathrm{Si}$ substrate, which was repeatedly exposed to oxygen plasma with 3 -s periods and then characterized by Raman spectroscopy after each plasma exposure dose. As expected, the $\mathrm{D}$ and $\mathrm{D}^{\prime}$ peaks, indicative of disorder, begin to rise and become more prominent with increased plasma exposure time, while conversely the twodimensional (2D) peak weakens and broadens ${ }^{49}$. Interestingly, when Raman spectra were obtained from the suspended graphene, the $\mathrm{D}$ peak appeared at shorter exposure times and the peak intensity ratios were different from those in the supported one as shown in Fig. 2b. The peak intensity ratios of D-G peaks $(\mathrm{I}(\mathrm{D}) / \mathrm{I}(\mathrm{G}))$, 2D-G peaks $(\mathrm{I}(2 \mathrm{D}) / \mathrm{I}(\mathrm{G}))$ and $\mathrm{D}-\mathrm{D}^{\prime}$ peaks $\left(\mathrm{I}(\mathrm{D}) / \mathrm{I}\left(\mathrm{D}^{\prime}\right)\right)$ for supported graphene are plotted in Fig. 2c. It is evident that $\mathrm{I}(\mathrm{D}) / \mathrm{I}(\mathrm{G})$ increases with plasma time until it reaches a maximum value of $\sim 4$ at $\sim 27 \mathrm{~s}$ and then decreases. On the other hand, $\mathrm{I}(2 \mathrm{D}) / \mathrm{I}(\mathrm{G})$ exhibited a slow decrease at the initial stage and then an abrupt decrease around $20 \mathrm{~s}$; such behaviours a

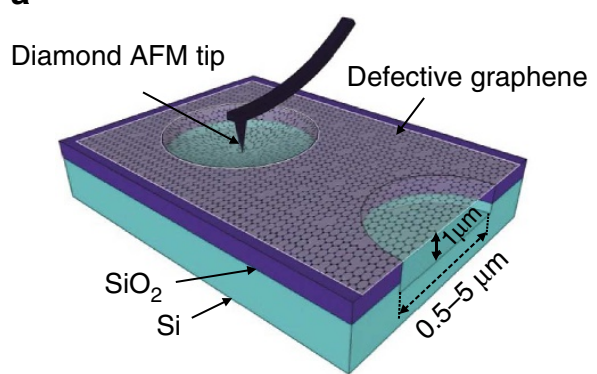

b

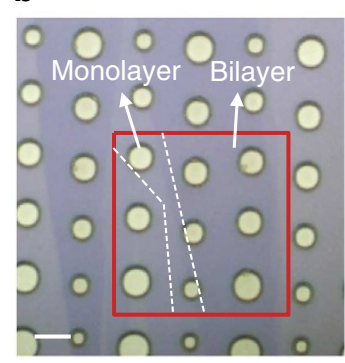

C

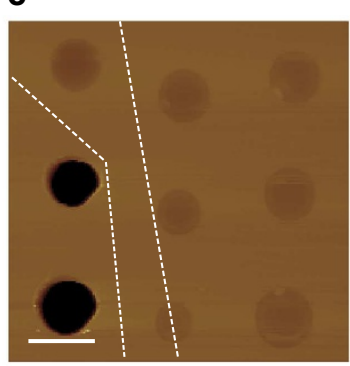

Figure 1 | Nanoindentation experiments and sample preparation. (a) Schematic representation of atomic force microscopy (AFM) nanoindentation test on suspended graphene sheets with defects. Graphene sheet is suspended over a hole with diameters ranging from 0.5 to $5 \mu \mathrm{m}$ and depth of $\sim 1 \mu \mathrm{m}$. (b) Optical micrograph of exfoliated graphene sheets suspended over holes. White-dashed line indicates the boundary of each layer. (c) Non-contact mode AFM image of suspended graphene sheet obtained from the red square box region marked in (b). Scale bars, $3 \mu \mathrm{m}$. 

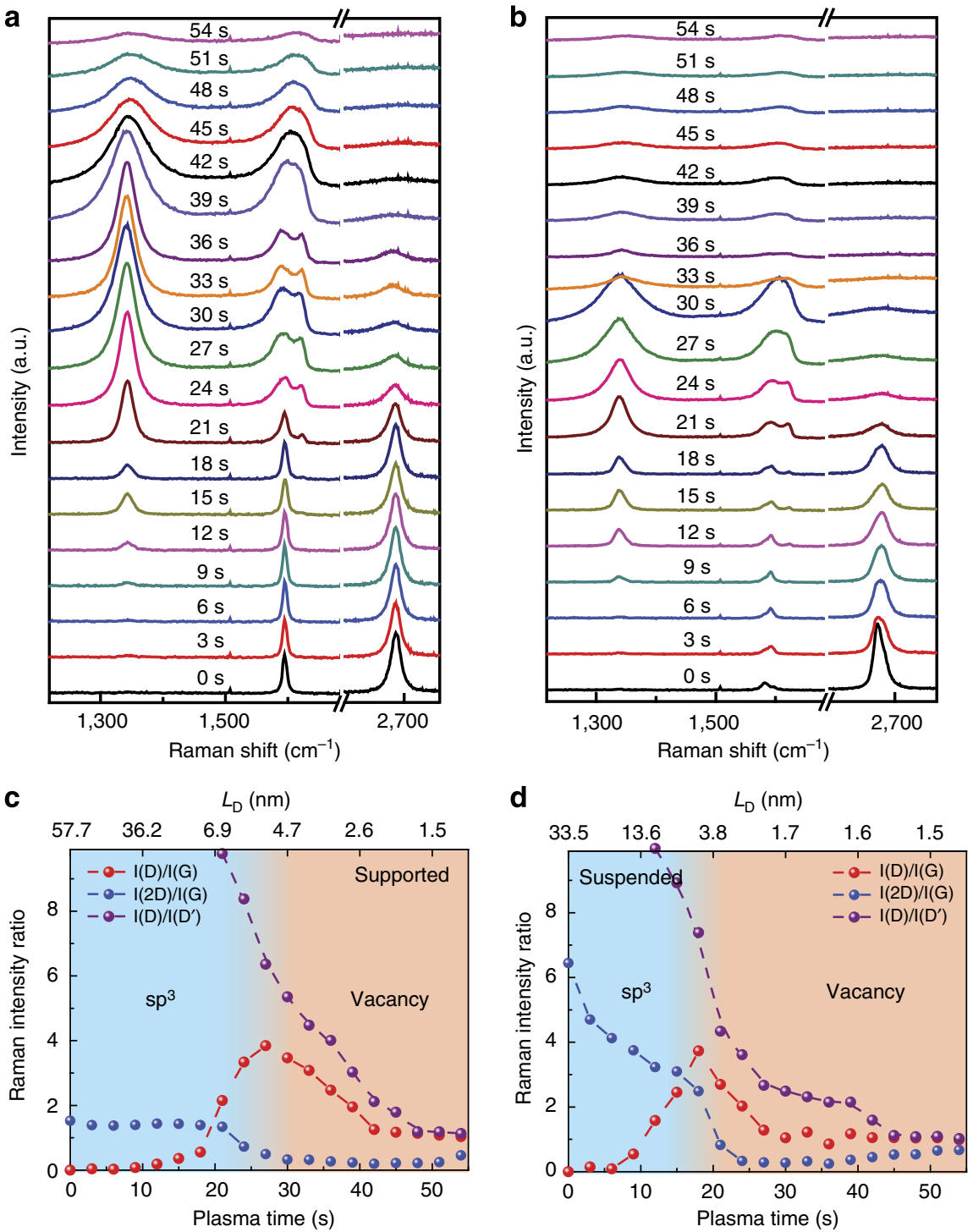

Figure 2 | Evolution of Raman spectra as a function of sheet defectiveness. Raman spectra of (a) supported graphene and (b) suspended graphene samples exposed to oxygen plasma for various numbers of 3-s steps. The evolution of D, G and 2D peaks can be observed as the plasma time exposure increases. Variation of Raman peak intensity ratios of (c) supported graphene and (d) suspended graphene samples as a function of plasma exposure time. For supported graphene, the I(D)/I(G) ratio increases with plasma exposure time, and then reaches a maximum around $27 \mathrm{~s}$ and drops back down, while the $I(2 D) / I(G)$ ratio shows a sudden drop around $20 \mathrm{~s}$. The suspended graphene exhibits a similar trend. Note that higher I(2D)/I(G) is observed in suspended graphene and maximum of $I(D) / I(G)$ is obtained at shorter plasma exposure time of $20 \mathrm{~s}$, indicating formation of more defects in the suspended graphene at the same plasma exposure time compared with supported graphene. The calculated average distance between defects $\left(L_{D}\right)$ is plotted as top $x$ axis. Noted that $L_{D}$ is indicated at the corresponding plasma exposure time without any linear relationship. According to I(D)/I(D') intensity ratio ${ }^{49}$ and defect type, the Raman maps of $\mathbf{c}$ and $\mathbf{d}$ are divided into two regimes of $s p^{3}$-type and vacancy-type defects. These regimes are indicated by blue and brown areas.

are also observed in the defective graphene induced by other etching techniques ${ }^{45-49}$. Although the Raman spectra (Fig. 2b) and peak intensity ratio (Fig. $2 \mathrm{~d}$ ) of the suspended graphene shows different values at the same plasma exposure time from those of the supported one, they follow a similar trend (Supplementary Fig. 2). Note that these differences in Raman spectra results between supported and suspended graphene sheets may be attributed to the substrate effect on Raman intensity in the supported graphene ${ }^{48}$, presence of pre-stress ${ }^{2}$ and etching of both sides ${ }^{50}$ in the suspended graphene.

It has been reported by Eckmann et al. ${ }^{49}$ that the $\mathrm{I}(\mathrm{D}) / \mathrm{I}\left(\mathrm{D}^{\prime}\right)$ intensity ratio can be used to discriminate between $s p^{3}$-type defects, in which an oxygen atom binds to the graphene and vacancy-type defects. Therefore, we categorize the defects ${ }^{49}$ as being predominantly $s p^{3}$-type (partial oxidation, $\mathrm{I}(\mathrm{D}) / \mathrm{I}\left(\mathrm{D}^{\prime}\right)>7$ ) or predominantly vacancy type $\left(\mathrm{I}(\mathrm{D}) / \mathrm{I}\left(\mathrm{D}^{\prime}\right)<7\right)$ as shown in Fig. 2c,d. As the crossover between these two regimes is gradual, the boundary is indicated by the blurred colour in Fig. 2c,d. The crossover region also correlates well to the point at which the $I(D) / I(G)$ intensity ratio achieves a maximum for both the suspended and supported graphene. Our molecular dynamics (MD) simulation results, which will be discussed in detail later, also confirmed the similar evolution of $s p^{3}$-type and vacancy-type defects with increasing plasma time. To measure the defect density, an average distance between defects $\left(L_{\mathrm{D}}\right)$, which is used as top $x$ axis in Fig. $2 \mathrm{c}$,d, was calculated using the $\mathrm{I}(\mathrm{D}) / \mathrm{I}(\mathrm{G})$ intensity ratio, as reported ${ }^{46,48}$ (see Supplementary Discussion for details). Note that for both supported and suspended graphene, 
a

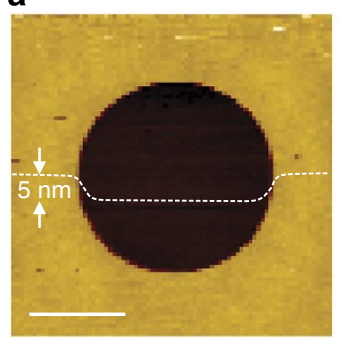

b

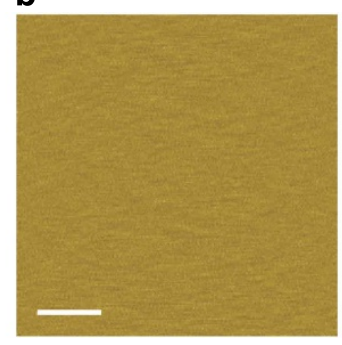

c

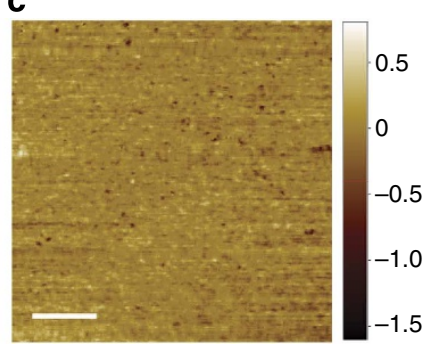

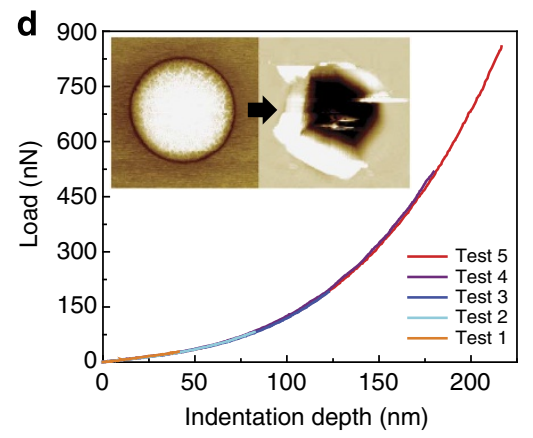

Figure 3 | Characterization of defective graphene by AFM and nanoindentation. (a) AFM image of a graphene sheet fully covering a hole. Highresolution AFM images of suspended graphene sheet (b) before and (c) after oxygen plasma exposure of $55 \mathrm{~s}$. It is obvious that the plasma treatment leaves the surface pock-marked with a multitude of nanopores that are several $\mathrm{nm}$ in size (the dark spots in the image represent the nanopores). (d) Typical force versus displacement curves of AFM nanoindentation test for defective graphene exposed to oxygen plasma for $30 \mathrm{~s}$. Tests are repeated at increasing indentation depths until the sample breaks. The curves fall on top of each other (no hysteresis), which indicates no significant sliding or slippage between the graphene membrane and the substrate. The AFM images in the inset of $\mathbf{d}$ show a graphene sheet before and after fracture. Scale bars, $1 \mu \mathrm{m}(\mathbf{a}) ; 100 \mathrm{~nm}(\mathbf{b}, \mathbf{c})$.

the $\mathrm{I}(\mathrm{D}) / \mathrm{I}(\mathrm{G})$ intensity ratio increased and reached a maximum at $L_{\mathrm{D}} \sim 5 \mathrm{~nm}$, and then decreased (Supplementary Fig. 2g). To further characterize the types of defects generated by oxygen plasma, high-resolution AFM imaging was performed with an ultra-sharp diamond-like carbon tip as shown in Fig. 3a-c. The membrane appears as a continuous sheet with no pores before plasma etching (Fig. 3a,b). After $55 \mathrm{~s}$ plasma etching (Fig. 3c), AFM imaging reveals extended defects, that is, nanopores, which are of the order of several $\mathrm{nm}$ in diameter (indicated by black dots in Fig. 3c), as reported elsewhere ${ }^{26,49}$. Suspended bilayer graphene sheets also showed a uniform distribution of nanopores (Supplementary Fig. 3), which confirms the homogenous etching of the graphene sheets by the oxygen plasma treatment.

AFM nanoindentation study. To study the evolution of mechanical properties with defect density, a series of samples was prepared and each was exposed to oxygen plasma for a given time (ranging from 0 to $55 \mathrm{~s}$ ), and was then characterized by Raman spectroscopy. Because of the variability of the oxygen plasma etching, the absolute etch time is not a reliable measure of the defect density. Consequently, the Raman parameters of $\mathrm{I}(\mathrm{D}) / \mathrm{I}(\mathrm{G})$ and $\mathrm{I}(2 \mathrm{D}) / \mathrm{I}(\mathrm{G})$ were used to quantify the degree of defectiveness of the graphene sheets. To measure mechanical properties, the suspended sheets were indented by AFM as reported previously ${ }^{2}$, using a diamond AFM tip with a radius of $80 \mathrm{~nm}$. Figure $3 \mathrm{~d}$ shows typical force-displacement curves for a defective graphene sheet exposed for $30 \mathrm{~s}$. AFM imaging of a defective graphene sheet after fracture (Fig. 3d, inset) indicates that fracture in the defective graphene occurs under the tip, as for pristine graphene. The 2D elastic modulus is determined by fitting the forcedisplacement response to a quasi-empirical polynomial form, containing linear and cubic deflection terms, as previously used for pristine graphene ${ }^{2}$ (see Supplementary Discussion for details).

Figure 4 a shows the evolution of the $2 \mathrm{D}$ elastic modulus $\left(E^{2 \mathrm{D}}\right)$ as a function of the Raman parameters. Remarkably, $E^{2 \mathrm{D}}$ remains constant (within experimental uncertainty) over the entire $s p^{3}$ type defect region (that is, when $\mathrm{I}(\mathrm{D}) / \mathrm{I}(\mathrm{G})<1, \mathrm{I}(2 \mathrm{D}) / \mathrm{I}(\mathrm{G})>1$ and $\left.\mathrm{I}(\mathrm{D}) / \mathrm{I}\left(\mathrm{D}^{\prime}\right)>7\right)$, indicating that these defects do not appreciably change the stiffness, even at a spacing $L_{\mathrm{D}}$ of $\sim 5 \mathrm{~nm}$. Once the graphene crosses over into the vacancy-type defect region (that is, when $\mathrm{I}(\mathrm{D}) / \mathrm{I}(\mathrm{G})>1, \mathrm{I}(2 \mathrm{D}) / \mathrm{I}(\mathrm{G})<1$ and $\left.\mathrm{I}(\mathrm{D}) / \mathrm{I}\left(\mathrm{D}^{\prime}\right)<7\right), E^{2 \mathrm{D}}$ decreases with increasing defect density, reaching $\sim 30 \%$ of the stiffness of the pristine sheet at the maximum exposure time.

Compared with the elastic stiffness, the breaking load of Fig. $4 \mathrm{~b}$ shows greater sensitivity to defects regardless of type. To calculate the breaking strength of defective graphene from the breaking load, we used the nonlinear finite element method based on $a b$ initio density functional theory calculations (see Supplementary Discussion). This analysis yields a strength of $\sim 35 \mathrm{~N} \mathrm{~m}^{-1}$ for pristine graphene, consistent with previous measurements ${ }^{3}$. Because of the highly nonlinear relationship between strength and breaking load, the approximately two-fold decrease in breaking load observed at an $\mathrm{I}(\mathrm{D}) / \mathrm{I}(\mathrm{G})$ ratio of $\sim 1.0$ corresponds to a decrease in strength of only $\sim 14 \%$, to $\sim 30 \mathrm{~N} \mathrm{~m}^{-1}$ (Supplementary Fig. 4). This is a central finding of our study: graphene can maintain a large fraction of its pristine strength and stiffness in the presence of a high density of $s p^{3}$-type defects. These results can help guide the rational design of graphene-based composite materials because one can now quantify the trade-off that exists between defects (for example, because of crosslinking or processing) and mechanical properties (such as 2D elastic modulus and strength). Once the graphene passes into the vacancy-type region, the linear elastic modulus (and presumably the higher-order elastic moduli as well) changes. Therefore, the nonlinear finite element method $^{3}$ cannot be applied in this regime. For a rough estimate, we note that in the linear elastic approximation, strength varies as $\left(F_{\mathrm{b}} E^{2 D}\right)^{0.5}$, where $F_{\mathrm{b}}$ is the breaking $\operatorname{load}^{2}$. In this simple model, the breaking strength at the highest plasma exposure time in our experiments is roughly four times smaller than that of pristine graphene.

\section{Discussion}

The mapping of the mechanical properties of defective graphene (Fig. 4a,b) to their Raman spectra indicates that, for $\mathrm{I}(\mathrm{D}) / \mathrm{I}(\mathrm{G})<1$ and $\mathrm{I}(2 \mathrm{D}) / \mathrm{I}(\mathrm{G})>1$ (that is, in the $s p^{3}$-type defect regime), the elastic stiffness of defective graphene is not significantly diminished in comparison with its pristine counterpart. This is surprising, since an $\mathrm{I}(\mathrm{D}) / \mathrm{I}(\mathrm{G})$ ratio of 1 is generally considered to represent a relatively high state of defectiveness in graphene. For instance, $\mathrm{I}(\mathrm{D}) / \mathrm{I}(\mathrm{G})$ values of $\sim 1.0$ are typical of graphene that is synthesized by chemical or thermal reduction of GO. Such reduced GO (RGO) sheets are known to offer significantly lower electrical $^{8}$ and thermal conductivity ${ }^{51}$ compared with pristine graphene owing to their defective state. By contrast, for mechanical properties, we find that graphene is capable of tolerating defects and retaining its ultra-high stiffness properties. As compared with the stiffness, the breaking force (Fig. 4b) of graphene shows greater sensitivity to defects. Nevertheless, owing to the nonlinear elastic behaviour of graphene (Supplementary 

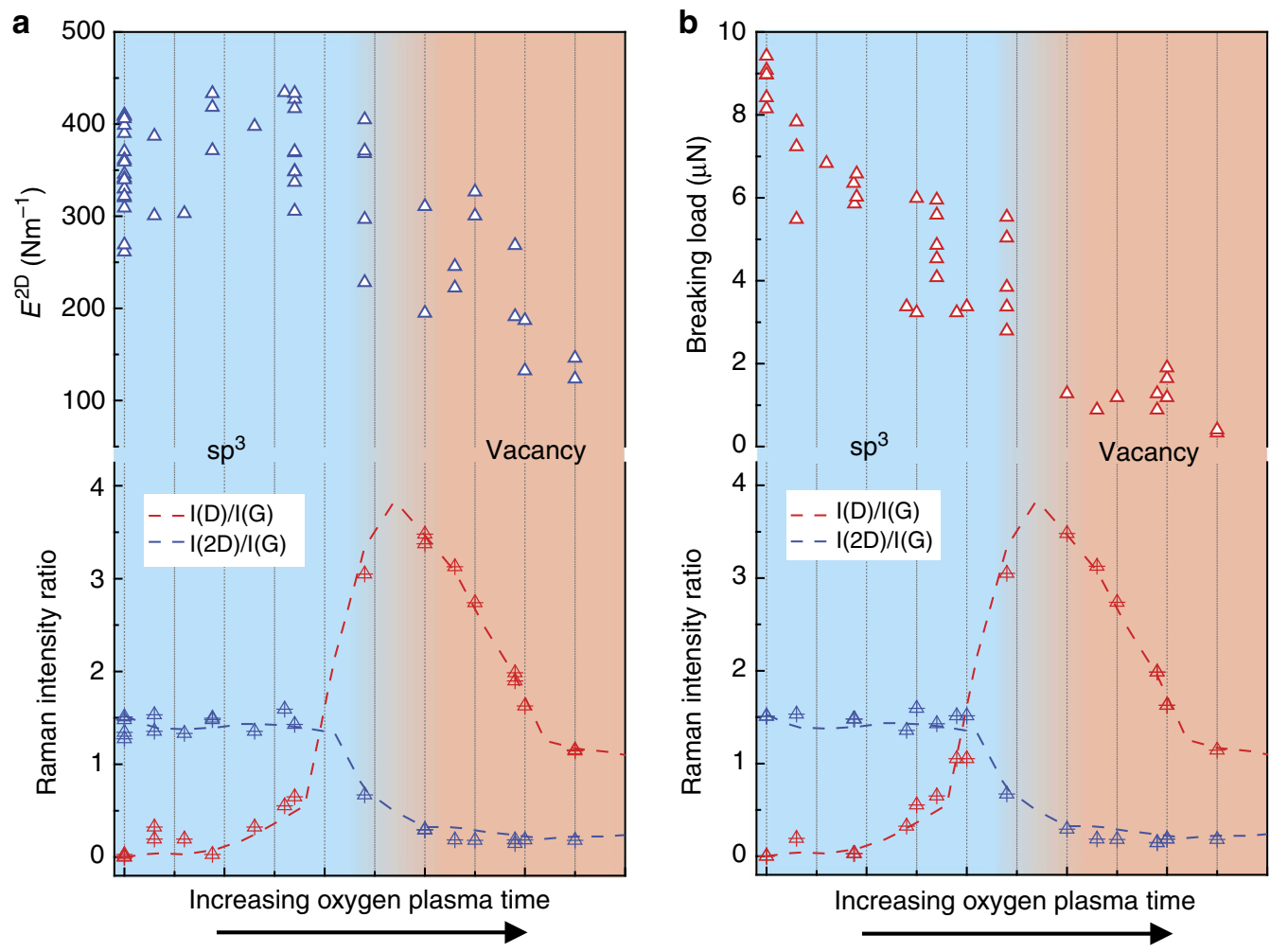

Figure 4 | Measurement of elastic stiffness and breaking load of defective graphene. Measured mechanical properties, (a) 2D elastic modulus and (b) breaking load of the defective graphene as a function of the Raman parameters of I(D)/I(G) and I(2D)/I(G) measured at increasing plasma times. Two regimes of $s p^{3}$-type and vacancy-type defects are indicated by blue and brown areas. In the $s p^{3}$-type regime (I(D)/I(G) ratio increases to $\left.\sim 4.0\right)$, the elastic stiffness was maintained but then dropped precipitously in the vacancy-defect regime. By contrast, the breaking load continuously decreased in both the $s p^{3}$-type and vacancy-type defect regimes.

Fig. 4), the strength of defective graphene is only $\sim 14 \%$ smaller than its pristine counterpart in the $s p^{3}$-type defect regime. Our results provide a simple, non-contact and non-destructive approach to quantifying the mechanical properties of defective graphene samples. This involves measuring the intensities of the Raman $D$ and $2 D$ peaks with respect to the $G$ peak. Using the direct mapping between the Raman parameters and the measured 2D elastic modulus and breaking load that we report (Fig. 4a,b), it is now possible to determine in a quantitative yet non-destructive manner how the mechanical properties of graphene vary as a function of its defectiveness. This has important practical implications for the design of a broad range of graphene-based devices such as chemical sensors ${ }^{7,8}$, ultra capacitors ${ }^{9}$, transparent electrodes ${ }^{10,11}$, photovoltaic cells $^{12}$, biodevices ${ }^{13-15}$, nanoelectronics ${ }^{24}$, DNA-sequencing through nanopores ${ }^{25}$ and selective molecular sieving through the nanopores of suspended graphene $\mathrm{e}^{26}$.

In addition to the Raman study, we also carried out aberrationcorrected high-resolution TEM (AC-HRTEM) characterization (Fig. 5) of the defective graphene lattice at both low and high oxygen plasma times. Figure $5 \mathrm{a}$ is representative of a graphene sheet in the $s p^{3}$-defect regime $(\mathrm{I}(\mathrm{D}) / \mathrm{I}(\mathrm{G}) \sim 0.2$ and $\mathrm{I}(2 \mathrm{D}) /$ $\mathrm{I}(\mathrm{G}) \sim 1.5)$ while Fig. $5 \mathrm{~b}$ is typical of a graphene sample in the vacancy-defect regime $(\mathrm{I}(\mathrm{D}) / \mathrm{I}(\mathrm{G}) \sim 2.0$ and $\mathrm{I}(2 \mathrm{D}) / \mathrm{I}(\mathrm{G}) \sim 0.2)$. Both TEM images show the presence of polymer residue (associated with the transfer process ${ }^{3}$ onto the TEM grid, see Methods). However, it is evident that while Fig. 5b indicates an abundance of nanocavities (that is, etch pits) in the graphene lattice (representative of the vacancy-defect regime), Fig. 5a shows a contrasting absence of such cavities. The black dots (circled in Fig. 5a,b) are $s p^{3}$ point defects that correspond to oxygen adatoms $^{52}$. The upper and lower insets in Fig. 5a show experimentally obtained TEM image and the corresponding simulated image of an oxygen atom attached to $s p^{2}$ carbon. The settings (Cs: $0.005 \mathrm{~mm}$, Scherzer focus: $-53 \AA$, operated at $80 \mathrm{keV}$ ) in the simulated TEM image is identical to what was used in the experimental image (see Methods). These images confirm that $s p^{3}$ point defects in the form of oxygen adatoms are generated in the $s p^{3}$-defect regime, while with increasing plasma exposure carbon atoms are etched from the lattice, resulting in the formation of nanocavities or nanopores in the vacancy-defect regime. Energy dispersive spectroscopy analysis (Supplementary Fig. 5) of the graphene suspended over the holes of the TEM grid also confirmed a significant increase in the oxygen content even at short plasma times (in the $s p^{3}$-type defect regime) as compared with pristine graphene.

It should be noted that our AFM nanoindentation technique reveals only the spatially averaged mechanical properties of the defective graphene sheet; the indented area in our testing is at least $4,500 \mathrm{~nm}^{2}\left(67 \times 67 \mathrm{~nm}^{2}\right)$, which contains at least 86,550 carbon hexagons (that is, at least 17,3100 carbon-carbon bonds). However, a recent study ${ }^{53}$ that used topographical AFM imaging on wrinkled GO and wrinkled chemically reduced GO sheets revealed significant local heterogeneity in the in-plane elastic modulus of such chemically modified carbon nanomaterials. The elastic modulus of GO produced by the chemical oxidation of graphene sheets was found to vary between 0.42 and $0.11 \mathrm{TPa}$ owing to defect concentration or clustering of different functional groups. They determined the average elastic modulus for GO to be $\sim 0.23 \mathrm{TPa}$; this is in reasonable agreement with the samples with the highest plasma exposure (Fig. 4a) that we tested, which showed elastic modulus of $\sim 0.3 \mathrm{TPa}$. For chemical vapour- 
a

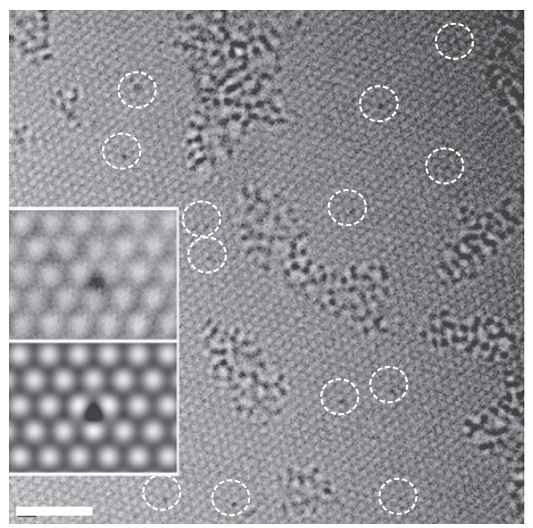

b

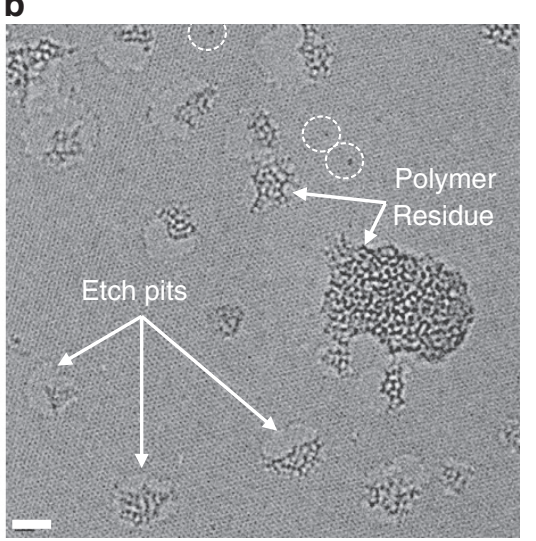

Figure 5 | AC-HRTEM characterization of defect structures. Images of a typical graphene sheet in (a) $s p^{3}$-type and (b) vacancy-type defect regime. Polymer residue associated with the transfer process onto the TEM grid is indicated by arrows. The defective graphene of the vacancy-type defect regime contains an abundance of nanocavities (that is, etch pits), while the defective graphene of the $s p^{3}$-type defect regime shows a contrasting absence of such cavities. The black dots circled with dashed lines in $\mathbf{a}$ and $\mathbf{b}$ are oxygen adatoms. The insets of $\mathbf{a}$ show the experimentally obtained TEM image (upper) and the corresponding simulated image (lower) of oxygen atoms bonded to carbon forming $s p^{3}$ point defects. Scale bars, $2 \mathrm{~nm}(\mathbf{a}, \mathbf{b})$.

deposited graphene, ref. 53 reported far less spatial heterogeneity in elastic properties with an averaged elastic modulus of $\sim 0.91 \mathrm{TPa}$, which shows good agreement with our result $(\sim 1 \mathrm{TPa})$ for pristine graphene (Fig. 4a). Another striking observation in ref. 53 was that the heterogeneities introduced during synthesis of $\mathrm{GO}$ were not removed in chemically reduced GO with the material still exhibiting significant spatial heterogeneity (the elastic modulus of chemically reduced GO varied between $\sim 0.82 \mathrm{TPa}$ and $\sim 0.36 \mathrm{TPa}$ ). This is important because mechanical failure is typically dictated by the weaker (or the more defective) regions. This is consistent with our results for breaking load (Fig. 4b), which indicate a higher sensitivity of the failure load to defects as compared with the elastic modulus.

In addition to the aforementioned experiments and defect characterization studies, we also performed atomistic modelling using the MD simulator, LAMMPS (large-scale atomic/molecular massively parallel simulator). The reactive force field (ReaxFF) potential used in this study is specially trained for simulation of hydrocarbon oxidation ${ }^{54}$ and can simulate continuous bond formation and breakage ${ }^{55}$. In the simulations, oxygen atoms are placed on both sides of the graphene sheet (Fig. 6a) and kept stationary during equilibration. The graphene is equilibrated for $2 \mathrm{ps}$ in the isobaric-isothermal ensemble at $300 \mathrm{~K}$ and zero pressure in all directions with a Nose-Hoover thermostat for temperature control and a time step of $0.2 \mathrm{fs}$ (Fig. 6b). Much longer equilibration periods were run in separate tests to insure that 2 ps would sufficiently equilibrate the systems under the given conditions. Post-equilibration, random velocity vectors corresponding to $300 \mathrm{~K}$ are assigned to oxygen atoms (Fig. 6c). Microcanonical ensemble with a time step of $0.2 \mathrm{fs}$ is used to run the simulation since the local temperature rise due to collision of carbon and oxygen atoms has a vital role in driving the reactions. Therefore, no external heat bath (that is, thermostat) was used as in ref. 56. Note that the oxygen plasma cleaner used in the experiments generates a cold (or non-thermal) plasma. Since we are simulating cold plasma, the velocity that is given to the oxygen atoms is considered to be equivalent to room temperature $(300 \mathrm{~K})$. The simulation ends after the oxygen has completely etched away the entire carbon network (Fig. 6d).

We considered four different numbers of oxygen atoms: 750 , $1,000,1,500$ and 4,500 in the simulations corresponding to varying oxygen pressures. These simulations are representative and have been selected from a large pool of runs of stochastically similar systems. The number of carbon atoms in the graphene sheet was kept constant at 1,500. In the Supplementary Discussion, we plot the number of carbon atoms removed from the graphene lattice by the oxygen plasma and the number of chemisorbed oxygen atoms normalized by the total number of carbon atoms in the graphene lattice as a function of the plasma time (Supplementary Fig. 6). The result resembles a cumulative Weibull distribution with the following three distinct regimes: (1) incubation period, (2) an intermediate regime and (3) a terminal regime. The incubation period corresponds to before the chemisorption of oxygen sets in. The intermediate regime is when oxygen atoms attach to the graphene lattice and generate defects. The terminal regime corresponds to fast growth, percolation and coalescence of defects resulting in large cavities in the lattice. During the terminal regime the number of carbon atoms removed from the graphene lattice increases sharply culminating in the complete breakup of the graphene structure. The structures formed and evolved during the intermediate regime are of interest for this study.

Let us consider the case of 1,000 oxygen atoms in the simulation. For this case, the incubation period ends and intermediate regime begins at $\sim 200 \mathrm{ps}$, while the terminal region begins after $\sim 320 \mathrm{ps}$ of plasma time (Supplementary Fig. 6). The time period from 200 to $350 \mathrm{ps}$ was divided into 10 equal subintervals and 10 structures corresponding to the end of each time interval, and the initial graphene structure at $200 \mathrm{ps}$ were selected for uniaxial tensile testing. The tensile loading simulations were performed at $300 \mathrm{~K}$ using a Nose-Hoover thermostat for temperature control and a time step of $0.2 \mathrm{fs}$ while maintaining zero pressure in the lateral (that is, armchair) direction. The calculated $2 \mathrm{D}$ elastic stiffness and ultimate strength for these 11 structures are plotted in Fig. 6e. The results mirror our experimental observations in that the elastic stiffness does not degrade significantly up to $\sim 310 \mathrm{ps}$ (corresponding to $s p^{3}$-defect regime), after which we see a rapid loss in elastic stiffness in the terminal regime, which corresponds to the vacancy-defect regime of Fig. 4a. The MD simulations qualitatively capture the experimental observations and shed light onto how defects influence the mechanical properties of graphene. The strength predictions (Fig. 6e) show a gradual decrease in strength up to $\sim 310$ ps (in the $s p^{3}$-defect regime) followed by a much more precipitous drop in the vacancy-defect regime (at $350 \mathrm{ps}$ ), which is also consistent with our experimental observations. It should be 
a

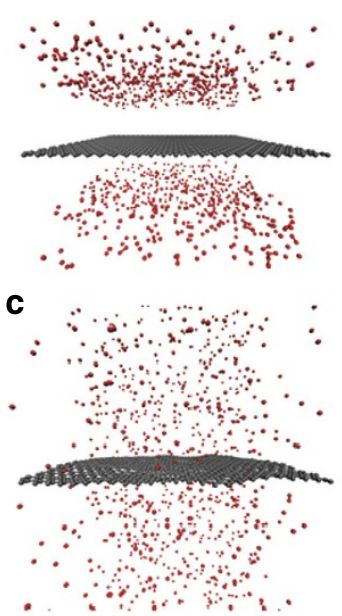

b
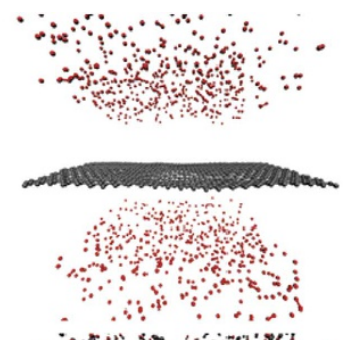

d

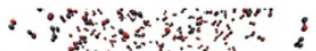

1

$\therefore-1+4=0$

1)

swetson

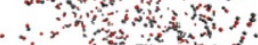

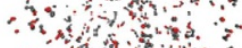

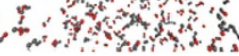

e

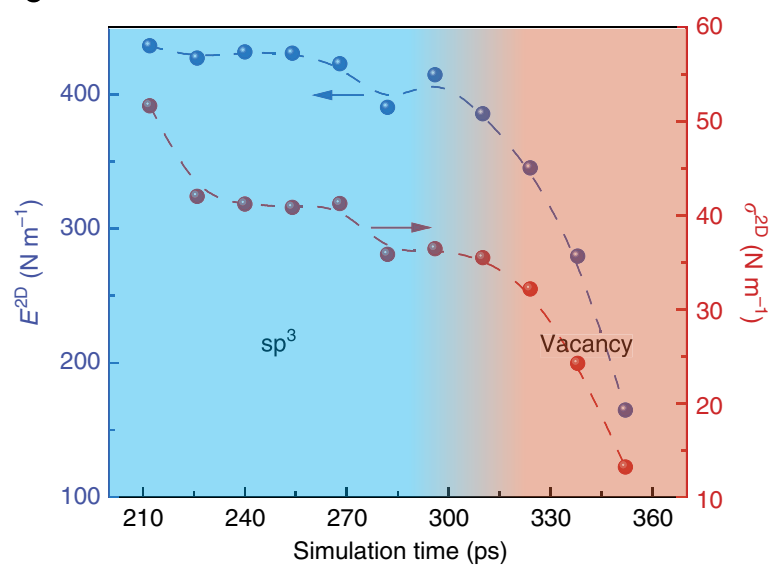

f

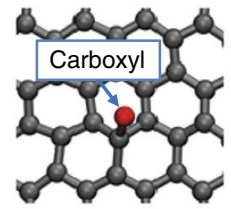

247.4 ps

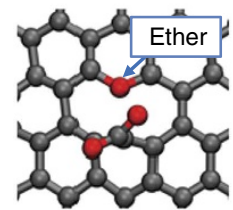

278.6 ps

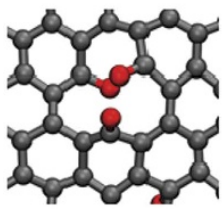

$311.8 \mathrm{ps}$

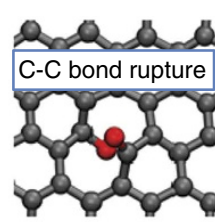

249.6 ps

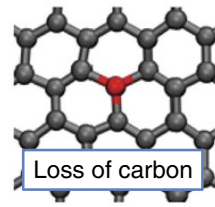

279.2 ps

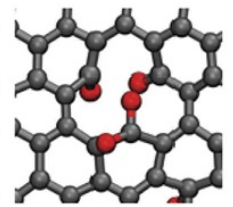

$317.8 \mathrm{ps}$

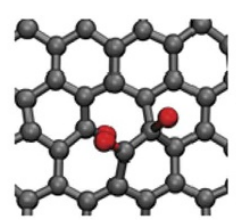

277.8 ps

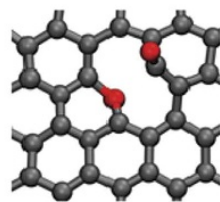

293.4 ps

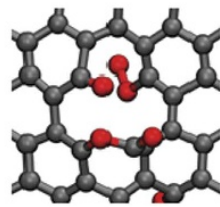

$319.8 \mathrm{ps}$

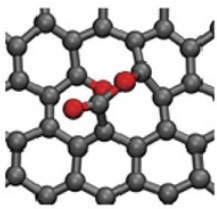

278 ps

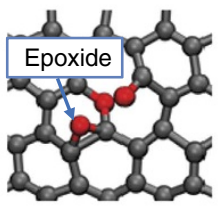

310.6 ps

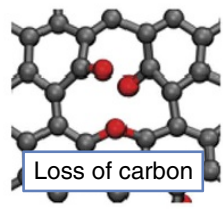

320.6 ps

g

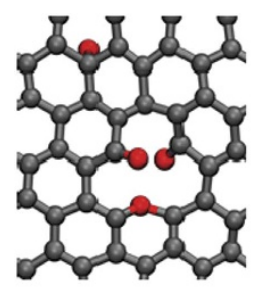

331.8 ps

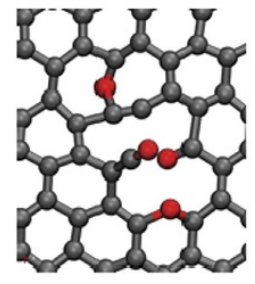

$338.4 \mathrm{ps}$

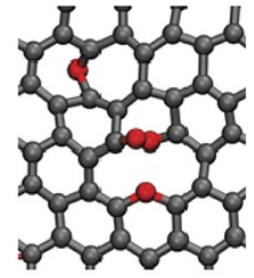

332.6 ps

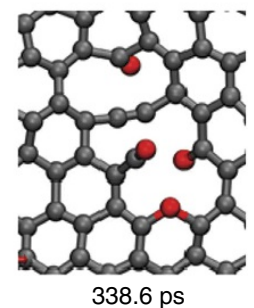

Figure 6 I MD simulations. (a) Simulation box with oxygen atoms (in red color) and carbon atoms (in grey colour) at time $=0$ ps. (b) At the end of initial equilibration of graphene sheet (time $=2 \mathrm{ps}$ ). (c) During the plasma treatment (time $=6.2 \mathrm{ps}$ ). (d) At the end of the simulation (time $=400 \mathrm{ps)}$.

(e) Calculated 2D elastic modulus and ultimate strength for the structures generated in the simulation with 1,000 initial oxygen atoms and 1,500 carbon atoms in the graphene sheet. (f) Series of snapshots at various times in the simulation showing chemisorption of oxygen as carbonyl, epoxide and ether groups and structural reorganization of the graphene lattice leading to the removal of carbon atoms in the form of carbon dioxide at 279.2 and 320.6 s. (g) An example of defect coalescence (at $338.6 \mathrm{ps)}$ initiated by an ether group.

noted that the predicted $2 \mathrm{D}$ elastic modulus for pristine graphene is $\sim 420 \mathrm{~N} \mathrm{~m}^{-1}$ (corresponds to $\sim 1.2 \mathrm{TPa}$ Young's modulus), which overestimates the elastic modulus of pristine graphene $(\sim 1 \mathrm{TPa})$. This is because the ReaxFF potential that was used in our study is not fully optimized (calibrated) for mechanical properties prediction. The choice of the ReaxFF potential in our work was dictated by its ability to simulate hydrocarbon oxidation as well as the continuous formation and breakage of bonds.

To explain why the elastic stiffness of the graphene sheet shows lower sensitivity to defects as compared with its breaking strength, we considered the structure of the chemisorbed oxygen and the processes of carbon atom removal from the graphene lattice. During the simulation, carbonyl groups are initially formed because of the chemisorption of oxygen (Fig. 6f). Increasing numbers of chemisorbed oxygen atoms then result in the formation of epoxide and ether groups. This functionalization of the graphene lattice with carbonyl, epoxide and ether groups involves rupture of some $\mathrm{C}-\mathrm{C}$ bonds that introduces discontinuities in the graphene lattice as indicated in Fig. 6f. Breaking of C-C bonds lowers the strength of the graphene sheet, which explains why the strength gradually drops in the experiments and in the simulations with the chemisorption of oxygen functional groups. Unlike strength, localized disruptions to the carbon bonding network does not significantly lower the elastic stiffness of the $2 \mathrm{D}$ graphene structure. However, the situation changes beyond $320.6 \mathrm{ps}$ of plasma time when significant numbers of carbon atoms are physically removed (Fig. 6f) from the graphene lattice. More importantly, as the density of defects increases (332.6 ps in Fig. 6g), adjacent defects coalesce to form bigger voids or extended cavities $(338.6 \mathrm{ps}$ in Fig. $6 \mathrm{~g}$ ). At this stage large numbers of carbon atoms are rapidly removed from the graphene lattice, which results in a precipitous drop in elastic stiffness and strength as the graphene structure 
breaks apart. The formation of nanocavities in the graphene lattice in the vacancy-defect regime is also corroborated by ACHRTEM imaging of the graphene sheet (Fig. 5b).

To summarize, over the entire $s p^{3}$-type defect region, the $2 \mathrm{D}$ elastic modulus and strength of graphene is relatively insensitive to defects, even at a spacing $L_{\mathrm{D}}$ of $\sim 5 \mathrm{~nm}$. This has important implications for design of graphene-based composite materials since it indicates that graphene can be covalently bonded to polymer matrices without sacrificing its reinforcing abilities. Only in the vacancy-defect regime, when the plasma begins to etch the graphene sheet and remove large numbers of carbon atoms, does the elastic modulus and strength of graphene begins to degrade significantly. The direct mapping between the Raman signature of defective graphene and its mechanical properties that we provide allows for a simple and non-destructive methodology to predict the 2D elastic modulus and breaking strength of defective graphene without actually testing it. These results could enable the rational design of graphene composites as well as mechanically stable graphene membranes for a variety of applications.

\section{Methods}

Sample preparation. To fabricate array of holes with various diameters of $0.5 \sim 5 \mu \mathrm{m}$, a Si wafer with $300 \mathrm{~nm} \mathrm{SiO}_{2}$-capping layer was patterned by conventional photolithography and reactive ion etching. The depth of hole is $\sim 1 \mu \mathrm{m}$ for indentation test. The graphene sheets were deposited on the patterned substrate by the well-established Scotch tape method ${ }^{2,18}$. A benchtop radio frequency oxygen plasma cleaner (Plasma Etch Inc., PE-50 XL, $100 \mathrm{~W}$ ) was utilized for introducing defects in graphene. To increase the controllability of defect generation, the chip was flipped and placed upside down on two carefully cleaned glass slides in the oxygen plasma chamber (Supplementary Fig. 1). For the AC-HRTEM imaging, the pristine samples were transferred to a TEM grid using poly(methyl methacrylate) transfer method ${ }^{3}$. After removal of poly(methyl methacrylate) by annealing, the samples were then exposed to oxygen plasma irradiation in the same manner.

Characterization of defects. The type and density of defects in the graphene sheets were characterized using Raman spectroscopy (Renishaw, invia with $532 \mathrm{~nm}$ wavelength laser and $0.7 \mu \mathrm{m}$ spot size). For high-resolution imaging of vacancytype defects, suspended graphene samples exposed by the oxygen plasma were carefully scanned by AFM with ultra-sharp diamond-like carbon tip (NT-MDT, NSG01_DLC) with a small tip radius of $<1 \mathrm{~nm}$. HRTEM instruments (JEOL, JEM$2100 \mathrm{~F}$ with double Cs correctors, operated at $80 \mathrm{keV}$ and JEOL, JED-2300T) were used for high-resolution imaging and energy dispersive spectroscopy, respectively. TEM image simulation of oxygen adatom (an oxygen atom attached to $s p^{3}$ carbon) was conducted using settings (Cs: $0.005 \mathrm{~mm}$, Scherzer focus: $-53 \AA$, operated at $80 \mathrm{keV}$ ) that are identical to that used in the experiments to acquire the images.

AFM nanoindentation. The precise nanoindentation on the suspended graphene was performed using AFM (Park Systems, XE-100). Before each indentation, the samples were scanned in non-contact mode to find graphene sheets fully covering a hole. After scanning, the diamond AFM tip (MicroStar Tech) was centred in the middle of the circular hole. Mechanical testing was performed using forcedisplacement mode. When non-trivial hysteresis was observed (presumably due to slippage of the graphene on the substrate), their corresponding data were discarded. The nonlinear force versus displacement curves obtained from the AFM nanoindentation tests were used to quantitatively determine the elastic stiffness and breaking load of defective graphene following the model described in refs 2 and 3.

MD simulations. LAMMPS was used for MD simulations as it has been extensively tested and successfully used for modelling solid, liquid and gaseous systems using different force fields and boundary conditions. For the purpose of atomistic modelling of graphene and its chemical reactions with oxygen, ReaxFF potential was used. The ReaxFF used in this study is specially trained for simulation of hydrocarbon oxidation ${ }^{54}$ and is able to bridge the gap between quantum chemical and empirical force field-based computational chemical methods ${ }^{54,55}$. In the initial state, the oxygen atoms are placed not closer than $1 \mathrm{~nm}$ from the graphene sheet surface. This distance is intentionally selected to be larger than the nearest neighbour cutoff distance $(0.45 \mathrm{~nm})$ to avoid initial interactions between oxygen atoms and the graphene sheet. To remove the in-plane pressure from graphene (pressure components along $x$ and $y$ directions), graphene is equilibrated for 2 ps in the isobaric-isothermal ensemble at $300 \mathrm{~K}$ and zero pressure in all directions with a Nose-Hoover thermostat (Tstart, Tstop of $300 \mathrm{~K}$ and Tdamp of $10 \mathrm{~K}$ ) for temperature control and a time step of $0.2 \mathrm{fs}$. After equilibration, random velocity vectors corresponding to $300 \mathrm{~K}$ are assigned to the oxygen atoms. The carbon atoms already have velocities corresponding to $300 \mathrm{~K}$. This is followed by running the simulation in the microcanonical ensemble with a time step of $0.2 \mathrm{fs}$ and without coupling the system to an external heat bath using a thermostat ${ }^{56}$. Additional information is provided in the Supplementary Discussion.

\section{References}

1. Geim, A. K. \& Novoselov, K. S. The rise of graphene. Nat. Mater. 6, 183-191 (2007).

2. Lee, C., Wei, X., Kysar, J. W. \& Hone, J. Measurement of the elastic properties and intrinsic strength of monolayer graphene. Science 321, 385-388 (2008).

3. Lee, G. H. et al. High strength chemical-vapor-deposited graphene and grain boundaries. Science 340, 1073-1076 (2013).

4. Stankovich, S. et al. Graphene-based composite materials. Nature 442, 282-286 (2006).

5. Rafiee, M. A. et al. Enhanced mechanical properties of nanocomposites at low graphene content. ACS Nano 3, 1-17 (2009).

6. Zandiatashbar, A., Picu, C. R. \& Koratkar, N. Control of epoxy creep using graphene. Small 8, 1676-1682 (2012).

7. Schedin, F. et al. Detection of individual gas molecules adsorbed on graphene. Nat. Mater. 6, 652-655 (2007).

8. Yavari, F., Castillo, E., Gullapalli, H., Ajayan, P. M. \& Koratkar, N. High sensitivity detection of $\mathrm{NO}_{2}$ and $\mathrm{NH}_{3}$ in air using chemical vapor deposition grown graphene. Appl. Phys. Lett. 100, 203120 (2012).

9. Stoller, M. D., Park, S., Zhu, Y., An, J. \& Ruoff, R. S. Graphene-based ultracapacitors. Nano Lett. 8, 3498-3502 (2008).

10. Yin, Z. et al. Electrochemical deposition of $\mathrm{ZnO}$ nanorods on transparent reduced graphene oxide electrodes for hybrid solar cells. Small 6, 307-312 (2010).

11. Gomez De Arco, L. et al. Continuous, highly flexible, and transparent graphene films by chemical vapor deposition for organic photovoltaics. ACS Nano 4, 2865-2673 (2010).

12. Wang, X., Zhi, L. \& Müllen, K. Transparent, conductive graphene electrodes for dye-sensitized solar cells. Nano Lett. 8, 323-327 (2008).

13. Ang, P. K., Chen, W., Wee, A. T. S. \& Loh, K. P. Solution-gated epitaxial graphene as pH sensor. J. Amer. Chem. Soc. 130, 14392-14393 (2008).

14. Cohen-Karni, T., Qing, Q., Li, Q., Fang, Y. \& Lieber, C. M. Graphene and nanowire transistors for cellular interfaces and electrical recording. Nano Lett. 10, 1098-1102 (2010).

15. Dong, X., Shi, Y., Huang, W., Chen, P. \& Li, L.-J. Electrical detection of DNA hybridization with single-base specificity using transistors based on CVDgrown graphene sheets. Adv. Mater. 22, 1649-1653 (2010).

16. McAllister, M. J. et al. Single sheet functionalized graphene by oxidation and thermal expansion of graphite. Chem. Mater. 19, 4396-4404 (2007).

17. Schniepp, H. C. et al. Functionalized single graphene sheets derived from splitting graphite oxide. J. Phys. Chem. B 110, 8535-8539 (2006).

18. Stankovich, S. et al. Stable aqueous dispersions of graphitic nanoplatelets via the reduction of exfoliated graphite oxide in the presence of poly(sodium 4-styrenesulfonate). J. Mater. Chem. 16, 155-158 (2006).

19. Kim, K. S. et al. Large-scale pattern growth of graphene films for stretchable transparent electrodes. Nature 457, 706-710 (2009).

20. Bae, S. et al. Roll-to-roll production of 30-inch graphene films for transparent electrodes. Nat. Nanotech. 5, 574-578 (2010).

21. Novoselov, K. S. et al. Two-dimensional atomic crystals. Proc. Natl Acad. Sci. USA 102, 10451-10453 (2005).

22. Park, S. \& Ruoff, R. S. Chemical methods for the production of graphenes. Nat Nanotech. 4, 217-224 (2009).

23. Kittel, C. Introduction to Solid State Physics 704 (John Wiley \& Sons, 2004).

24. Nourbakhsh, A. et al. Bandgap opening in oxygen plasma-treated graphene. Nanotech. 21, 435203 (2010).

25. Garaj, S. et al. Graphene as a subnanometre trans-electrode membrane. Nature 467, 190-193 (2010).

26. Koenig, S. P., Wang, L., Pellegrino, J. \& Bunch, J. S. Selective molecular sieving through porous graphene. Nat. Nanotech. 7, 728-732 (2012).

27. Fedorov, A. S. et al. Mobility of vacancies under deformation and their effect on the elastic properties of graphene. J. Exp. Theor. Phys. 112, 820-824 (2011)

28. Tapia, A., Peón-Escalante, R., Villanueva, C. \& Avilés, F. Influence of vacancies on the elastic properties of a graphene sheet. Comp. Marter. Sci. 55, 255-262 (2012).

29. Xiao, J. R., Staniszewski, J. \& Gillespie, J. W. Tensile behaviors of graphene sheets and carbon nanotubes with multiple Stone-Wales defects. Mater. Sci. Eng. A 527, 715-723 (2010).

30. Paci, J. T., Belytschko, T. \& Schatz, G. C. Computational studies of the structure, behavior upon heating and mechanical properties of graphite oxide. J. Phys. Chem. C 111, 18099-18111 (2007).

31. Khare, R. et al. Coupled quantum mechanical/molecular mechanical modeling of the fracture of defective carbon nanotubes and graphene sheets. Phys. Rev. B 75, 1-12 (2007).

32. Dettori, R., Cadelano, E. \& Colombo, L. Elastic fields and moduli in defected graphene. J. Phys. Condens. Matter 24, 104020 (2012). 
33. Wang, M. C., Yan, C., Ma, L., Hu, N. \& Chen, M. W. Effect of defects on fracture strength of graphene sheets. Comp. Mater. Sci. 54, 236-239 (2012).

34. Xiao, J. R., Staniszewski, J. \& Gillespie, J. W. Fracture and progressive failure of defective graphene sheets and carbon nanotubes. Compos. Struct. 88, 602-609 (2009).

35. Ansari, R., Ajori, S. \& Motevalli, B. Mechanical properties of defective singlelayered graphene sheets via molecular dynamics simulation. Superlattice Microst. 51, 274-289 (2012).

36. Pei, Q. X., Zhang, Y. W. \& Shenoy, V. B. A molecular dynamics study of the mechanical properties of hydrogen functionalized graphene. Carbon NY 48, 898-904 (2010)

37. Meyer, J. C. et al. Direct imaging of lattice atoms and topological defects in graphene membranes. Nano Lett. 8, 3582-3586 (2008).

38. Banhart, F., Kotakoski, J. \& Krasheninnikov, A. V. Structural defects in graphene. ACS Nano 5, 26-41 (2011).

39. Stone, A. J. \& Wales, D. J. Theoretical studies of icosahedral C60 and some related species. Chem. Phys. Lett. 128, 501-503 (1986)

40. Bonilla, L. \& Carpio, A. Graphene Simulation: Theory of Defect Dynamics in Graphene. Ch. 9, 167-182 (In Tech, 2011).

41. Bagri, A. et al. Structural evolution during the reduction of chemically derived graphene oxide. Nat. Chem. 2, 581-587 (2010).

42. Bagri, A., Grantab, R., Medhekar, N. V. \& Shenoy, V. B. Stability and formation mechanisms of carbonyl- and hydroxyl-decorated holes in graphene oxide. J. Phys. Chem. C 114, 12053-12061 (2010).

43. Suk, J. W., Piner, R. D., An, J. \& Ruoff, R. S. Mechanical properties of monolayer graphene oxide. ACS Nano 4, 6557-6564 (2010).

44. Kim, D. C. et al. The structural and electrical evolution of graphene by oxygen plasma-induced disorder. Nanotechnol. 20, 375703 (2009).

45. Cancado, L. G. et al. Quantifying defects in graphene via Raman spectroscopy at different excitation energies. Nano Lett. 11, 3190-3196 (2011).

46. Lucchese, M. M. et al. Quantifying ion-induced defects and Raman relaxation length in graphene. Carbon NY 48, 1592-1597 (2010).

47. Dresselhaus, M. S., Jorio, A., Souza Filho, G. \& Saito, R. Defect characterization in graphene and carbon nanotubes using Raman spectroscopy. Philos. T. Roy. Soc. A 368, 5355-5377 (2010).

48. Araujo, P. T., Terrones, M. \& Dresselhaus, M. S. Defects and impurities in graphene-like materials. Mater. Today 15, 98-109 (2012).

49. Eckmann, A. et al. Probing the nature of defects in graphene by Raman spectroscopy. Nano Lett. 12, 3925-3930 (2012).

50. Mathew, S. et al. Mega-electron-volt proton irradiation on supported and suspended graphene: a Raman spectroscopic layer dependent study. J. App. Phys. 110, 084309 (2011).

51. Balandin, A. A. Thermal properties of graphene and nanostructured carbon materials. Nat. Mater. 10, 569-581 (2011)

52. Gómez-Navarro, C. et al. Atomic structure of reduced graphene oxide. Nano Lett. 10, 1144-1148 (2010).
53. Kunz, D. A. et al. Space-resolved in-plane moduli of graphene oxide and chemically derived graphene applying a simple wrinkling procedure. $A d v$. Mater. 25, 1337-1341 (2013).

54. Chenoweth, K., Van Duin, A. C. T. \& Goddard, W. A ReaxFF reactive force field for molecular dynamics simulations of hydrocarbon oxidation. J. Phys. Chem. A 112, 1040-1053 (2008).

55. Mueller, J. E., Van Duin, A. C. T. \& Goddard, W. A. Development and validation of ReaxFF reactive force field for hydrocarbon chemistry catalyzed by nickel. J. Phys. Chem. C 114, 4939-4949 (2010).

56. Srinivasan, S. G. \& Van Duin, A. C. T. Molecular-dynamics-based study of the collisions of hyperthermal atomic oxygen with graphene using the ReaxFF reactive force field. J. Phys. Chem. A 115, 13269-13280 (2011).

\section{Acknowledgements}

N.K. and C.R.P. acknowledge the funding support from the US Office of Naval Research (Award Number: N000140910928) and the US National Science Foundation (Award Number: 1234641). N.K. also acknowledges support from the John A. Clark and Edward T. Crossan endowed chair professorship at the Rensselaer Polytechnic Institute. J.H. acknowledges support from AFOSR MURI Program on new graphene materials technology, FA9550-09-1-0705, and G.-H.L. acknowledges the support from Samsung-SKKU Graphene Center. We thank Ryan Cooper and Jeffrey W. Kysar for fruitful discussion.

\section{Author contributions}

A.Z. and G.-H.L. contributed equally to this work. A.Z. and G.-H.L. jointly designed the AFM, plasma and Raman characterization tests. A.Z., G.-H.L., S.L. and S.J.A. jointly prepared the samples, performed the AFM and Raman characterization tests, and analysed the data. A.Z. and N.M. designed and prepared the LAMMPS simulation set-ups and post-processing scripts. M.T. and T.H. performed the HRTEM, energy dispersive spectroscopy characterization and analysis. N.K. and C.R.P. supervised the study at Rensselaer Polytechnic Institute. J.H. supervised the study at Columbia University. A.Z., G.-H.L., C.R.P., J.H. and N.K. wrote the manuscript.

\section{Additional information}

Supplementary Information accompanies this paper at http://www.nature.com/ naturecommunications

Competing financial interests: The authors declare no competing financial interests

Reprints and permission information is available online at http://npg.nature.com/ reprintsandpermissions

How to cite this article: Zandiatashbar, A. et al. Effect of defects on the intrinsic strength and stiffness of graphene. Nat. Commun. 5:3186 doi: 10.1038/ncomms4186 (2014) 